FERMILAB-CONF-08-218-E

June 30, 2008

\title{
Searches for Large Extra Dimensions at the Tevatron
}

\author{
Vyacheslav Krutelyov $^{1}$ for the CDF and the DØ Collaborations * \\ 1- University of California at Santa Barbara - Dept of Physics \\ Santa Barbara CA 93106-9530 - U.S.A.
}

June 30, 2008

\begin{abstract}
The presence of extra dimensions can be probed in high energy collisions via the production or exchange of gravitons. The former corresponds to signatures with missing energy while the latter corresponds to modifications of the final state spectra. Here I review results of analyses performed by the CDF and $\mathrm{D} \varnothing$ Collaborations on $p \bar{p}$ collisions at $\sqrt{s}=1.96 \mathrm{TeV}$ in signatures sensitive to large extra dimensions. These include analyses of $\gamma+\mathbb{H}_{T}$ and jet $+\mathbb{H}_{T}$ as signatures of graviton production as well as analyses of dilepton and diboson final states sensitive to graviton exchange.
\end{abstract}

\section{Introduction}

The standard model of particle physics (SM) does not account for gravitational interactions. A number of theoretical models beyond the SM naturally include gravity and require the existence of extra dimensions where only gravity propagates freely. In particular, a model proposed in Ref. [1] assumes there are large extra dimensions (LED) compactified on a sub-millimeter scale $R$. In this model gravity can become strong at the TeV scale, which effectively solves the hierarchy problem present in the SM. For $n$ extra dimensions the four-dimensional Planck mass $M_{\mathrm{Pl}}$ is related to the $4+n$-dimensional fundamental scale $M_{D}$ by $M_{\mathrm{Pl}}^{2}=8 \pi M_{D}^{2}\left(M_{D} R\right)^{n}$. For $M_{D}$ in the TeV range $R$ is of the order of $0.1 \mathrm{~mm}(10 \mathrm{fm})$ for $n=2(6)$. To solve the hierarchy problem $M_{D}$ should not be too large, which rules out $n=1$. Tests of the Newton's law constrain $R$ to be below $37 \mu \mathrm{m}$ for $n=2[2]$. Other constraints come from astrophysics (up to $n=3$ ) and from high energy colliders [2].

The cross sections of the LED processes depend on $M_{D}$ and can be at levels detectable at the Tevatron [3]. In $p \bar{p}$ collisions the graviton can be produced in $q \bar{q} \rightarrow g G, q g \rightarrow q G$, or

${ }^{*}$ Work supported by the U.S. Department of Energy under contract No. DE-AC02-07CH11359. 
$g g \rightarrow g G$ corresponding to a jet $+\mathbb{E}_{T}$ final state, and $q \bar{q} \rightarrow \gamma G$ corresponding to a $\gamma+\mathbb{E}_{T}$ detector signature [4]. Graviton exchange can be studied in a range of $2 \rightarrow 2$ processes with the best sensitivity in final states with two leptons, photons, or $Z$ bosons.

In this review [5] I first describe searches for graviton production in the $\gamma+\mathbb{E}_{T}$ and jet $+\mathbb{F}_{T}$ signatures reported by CDF and in the $\gamma+\mathbb{F}_{T}$ signature reported by D $\varnothing$. Then I briefly describe searches sensitive to graviton exchange in dimuon, dielectron and diphoton final states reported by $\mathrm{D} \varnothing$ and in $Z Z$ final states by both CDF and $D \varnothing$.

\section{$2 \mathrm{CDF}$ and $\mathrm{D} \emptyset$ detectors}

A detailed description of the CDF and the D $\varnothing$ detectors can be found in Ref. [6, 7]. The vertexing and the tracking detectors surrounding the interaction region are used to reconstruct charged particle trajectories. Further out are the calorimeters with electromagnetic and hadronic longitudinal segmentation used to identify and measure photons, electrons, and jets. Outside the calorimeter are the muon detectors used to identify muons from the collisions and cosmic rays.

In analyses of $\gamma+\mathbb{F}_{T}$ events one of the major backgrounds is from cosmic ray muons misidentified as photons. The detector features used to reject this background are: photon pointing available at $\mathrm{D} \varnothing$ allowing the reconstruction of direction of the photon from the longitudinal segmentation of the electromagnetic calorimeter [8]; photon timing available at CDF from the EMTiming system [9] providing the measurement of the time of the energy deposit and allowing to suppress cosmic-ray background by a factor of 20 or more.

\section{Searches for graviton production}

The production of gravitons in hadron collisions is probed in events where the final-state hadronic jet or photon recoils against the graviton, which is not detected and results in $\mathbb{E}_{T}$. Searches in both the jet $+\mathbb{E}_{T}$ and the $\gamma+\mathbb{E}_{T}$ require exclusive signatures: only a jet or a photon is in the event with a veto on the presence of other objects. These searches have similar backgrounds. The dominant and only irreducible background is production of $Z \rightarrow \nu \nu$ in association with a photon or a jet.

A search for LED in jet $+\mathbb{F}_{T}$ events has been performed by CDF using $1.1 \mathrm{fb}^{-1}$ of data, updating the analysis in Ref. [10]. Events with high $\mathbb{F}_{T}$, a high- $E_{T}$ jet and no second jet with $E_{T}>60 \mathrm{GeV}$ are selected. All major backgrounds are estimated in a data-driven way. The contributions from $Z \rightarrow \nu \nu$ and from $W \rightarrow \ell \nu$, where the lepton is lost, are estimated using measured $Z \rightarrow \ell \ell$ and $W \rightarrow \ell \nu$ events. After an a priori optimization for

\begin{tabular}{|l|c|}
\hline Background & Events \\
\hline$Z \rightarrow \nu \nu$ & $388 \pm 30$ \\
$W \rightarrow \ell \nu$ & $362 \pm 17$ \\
Multi-jet & $23 \pm 20$ \\
$\gamma+$ jet & $17 \pm 5$ \\
Non-collision & $10 \pm 10$ \\
Total predicted & $808 \pm 62$ \\
Data observed & 809 \\
\hline
\end{tabular}

Table 1: The numbers of expected background and observed events in the jet $+\mathbb{E}_{T}$ sample used by CDF. 
$E_{T}>150 \mathrm{GeV}$ and $\mathbb{E}_{T}>120 \mathrm{GeV}$ are selected. The summary of predicted and observed events is given in Table 1. The constraints on LED are given at the end of this section.

\begin{tabular}{|l|c|c|}
\hline Background & CDF & DØ \\
\hline$Z \gamma \rightarrow \nu \nu \gamma$ & $24.8 \pm 2.8$ & $12.1 \pm 1.3$ \\
$W \rightarrow(e \rightarrow \gamma) \nu$ & $2.6 \pm 0.4$ & $3.8 \pm 0.3$ \\
$W \rightarrow(\mu / \tau \rightarrow \gamma) \nu$ & $1.0 \pm 0.2$ & - \\
$W \gamma \rightarrow \ell \nu \gamma$ & $5.0 \pm 1.4$ & $1.5 \pm 0.2$ \\
jet $\rightarrow \gamma$ & - & $2.2 \pm 1.5$ \\
$\gamma \gamma \rightarrow \gamma$ & $2.3 \pm 0.6$ & - \\
Non-collision & $9.8 \pm 1.3$ & $2.8 \pm 1.4$ \\
Total predicted & $46.3 \pm 3.0$ & $22.4 \pm 2.5$ \\
Observed data & 40 & 29 \\
\hline
\end{tabular}

Table 2: The numbers of expected background and observed events in the $\gamma+\mathbb{F}_{T}$ samples analyzed by CDF and D $\varnothing$. The contribution from jet $\rightarrow \gamma$ in CDF is included in $(W / \gamma) \gamma$ by virtue of the background estimation method.
Results for the $\gamma+\mathbb{F}_{T}$ signature in the Tevatron Run II have been recently reported by $\mathrm{CDF}$ in $2 \mathrm{fb}^{-1}$ of data and by $\mathrm{D} \varnothing$ in $1.05 \mathrm{fb}^{-1}$ of data [8]. Both analyses use events with high $\mathbb{H}_{T}$ and a high- $E_{T}$ photon in the central detector region with $|\eta|<1.1$ [4]. No jets with $E_{T}>15 \mathrm{GeV}$ are allowed in the event in order to suppress QCD backgrounds. Also, no high- $p_{T}$ track is allowed, suppressing contributions from leptonic $W$ and $Z$ decays: tracks with $p_{T}>10 \mathrm{GeV} / c$ (isolated with $p_{T}>6.5 \mathrm{GeV} / c$ ) are vetoed by $\mathrm{CDF}$ $(\mathrm{D} \varnothing)$. The contribution from cosmic rays is suppressed based on presence of hits in the muon detectors as well as using the photon pointing or the photon timing mentioned in Section 2.

The background contributions for both analyses are summarized in Table 2 . The number of events from $Z \rightarrow \nu \nu$ is estimated from simulation. The contribution from $W \rightarrow e \nu$, where the electron is identified as the photon, is extracted from the number of events with electrons passing all other requirements applied to the photon signal sample, scaled by the misidentification rate. Contributions from cosmic rays and jets misidentified as photons are estimated at D $\varnothing$ using the photon pointing method. The remaining backgrounds in the analysis by $\mathrm{D} \varnothing$ are estimated using simulation. The contribution from cosmics at CDF is estimated using events with the photon time significantly different from the collision time. The remaining backgrounds at CDF are from processes where an object is lost. Except for $W \rightarrow \tau \nu$, which is estimated from simulation, each of these backgrounds is given by the number of events in data with this object identified, scaled by the simulated rate for it to be lost. This approach implicitly includes contributions from a jet identified as a photon.

After an a priori optimization for the best sensitivity, events with a photon with $E_{T}>$ $90 \mathrm{GeV}$ and $\mathbb{F}_{T}>50(70) \mathrm{GeV}$ are selected in the CDF (DØ) signal sample. Event counts observed in the data are consistent with expectations from the backgrounds. The constraints on the LED model are summarized in Table 3. Since the sensitivity in the $\gamma+\mathbb{E}_{T}$ is similar to that in the jet $+\mathbb{F}_{T}$ mode, the constraints can be improved in a combination. The result of this combination using the CDF data is shown in Table 3. Depending on the number of extra dimensions, the sensitivity to LED at the Tevatron is comparable to or better than that from the LEP experiments [11]. 


\begin{tabular}{|l|c|c|c|c|c|}
\hline Source & LEP & D $\varnothing$ & \multicolumn{3}{|c|}{ CDF } \\
$n$ & $\gamma+\mathbb{H}_{T}$ & $\gamma+\mathbb{F}_{T}$ & jet $+\mathbb{F}_{T}$ & $\gamma+\mathbb{H}_{T}$ & combined \\
\hline 2 & 1600 & 921 & 1310 & 1080 & 1400 \\
3 & 1200 & 877 & 1080 & 1000 & 1150 \\
4 & 940 & 848 & 980 & 970 & 1040 \\
5 & 770 & 821 & 910 & 930 & 980 \\
6 & 660 & 810 & 880 & 900 & 940 \\
\hline
\end{tabular}

Table 3: Lower limits on $M_{D}$ in $\mathrm{GeV} / c^{2}$ at $95 \%$ C.L. for $n$ from 2 to 6 observed in the jet $+\mathbb{F}_{T}$ and $\gamma+\mathbb{E}_{T}$ signatures and the combination of the two at CDF, and in the $\gamma+\mathbb{E}_{T}$ signature at $\mathrm{D} \varnothing$, along with the constraints from the LEP experiments [11]. Constraints provided by DØ for $n$ above 6 can be found in [8].

\section{Searches for virtual graviton exchange}

In hadron collisions the processes most sensitive to virtual graviton exchange in LED are those with two leptons (electrons or muons), two photons, or two $Z$ bosons in the final state, where it is possible to reconstruct the full kinematics of the final state with high precision. The value of the graviton exchange amplitude depends on the cutoff energy $\Lambda$, presumably of order $M_{D}$ [3]. The cross section is proportional to $\Lambda^{-8}$.

A search for LED in the dimuon final state has been reported by D $\varnothing$ using $200 \mathrm{pb}^{-1}$ of data [12]. The sample is comprised of events with two opposite charge muons with $p_{T}$ above $15 \mathrm{GeV} / c$ and both muons passing additional quality and isolation requirements. The dilepton invariant mass and the scattering angle in the dilepton center of mass are analyzed simultaneously to improve sensitivity to the exchange of a graviton (spin 2 particle). A similar analysis using a di-em final state where no discrimination is made between electrons and photons has been performed at $\mathrm{D} \varnothing$ with $240 \mathrm{pb}^{-1}$ of data [13]. In this case events were selected if they had two em objects with $E_{T}>25 \mathrm{GeV}$ passing quality and isolation requirements. No excess over the expected backgrounds is observed. The constraints on the parameter $\Lambda$ are in the range of 1 to $2 \mathrm{TeV}$ depending on the theoretical parameters.

Both CDF and $\mathrm{D} \varnothing$ have recently reported on searches for $p \bar{p} \rightarrow Z Z$ production in a four-lepton final state $[14,15]$. The observed number of events is consistent with the SM expectations. Although no constraint has been provided on LED by either experiment, based on Ref. [16] the constraint on $\Lambda$ is expected to be in the range of 1.5 to $2.5 \mathrm{TeV}$.

\section{Summary}

Searches for LED in signatures with direct graviton production and those with virtual graviton exchange are explored by the CDF and the D $\varnothing$ experiments. No evidence of LED has been seen. Additional $p \bar{p}$ collision data currently produced at the Tevatron is expected to give more insight about the possible presence of large extra spacial dimensions. 


\section{Acknowledgements}

I would like to acknowledge the funding institutions supporting the CDF and D $\varnothing$ Collaborations. The full list of agencies can be found in, e.g, $[6,7]$.

\section{References}

[1] N. Arkani-Hamed, S. Dimopoulos and G. R. Dvali, Phys. Lett. B 429, 263 (1998) [arXiv:hep-ph/9803315].

[2] W. M. Yao et al. [Particle Data Group], J. Phys. G 33, 1 (2006).

[3] G. F. Giudice, R. Rattazzi and J. D. Wells, Nucl. Phys. B 544, 3 (1999) [arXiv:hep-ph/9811291].

[4] The polar coordinate system is used with an origin at the center of a detector and the $z$-axis $(\theta=0)$ along the proton beam. The pseudorapidity $\eta$ is defined as $\eta=-\ln [\tan (\theta / 2)]$. The transverse momentum $p_{T}$ is defined as $p_{T}=p \cdot \sin \theta$, where $p$ is the particle's momentum. The transverse energy is defined as $E_{T}=E \cdot \sin \theta$, where $E$ is the energy measured by the calorimeter. The missing $E_{T}\left(\mathbb{E}_{T}\right)$ is defined as $\overrightarrow{E_{T}}=-\sum_{i} E_{T}^{i} \hat{n}_{i}$, where $i$ is the index of a calorimeter tower (a segmentation unit in $\eta-\phi$ ) and $\hat{n}_{i}$ is a unit vector perpendicular to the beam axis pointing to the tower from the origin.

[5] Slides:

http: //indico. cern. ch/contributionDisplay py? contribId=104\&sessionId=15\&conf Id=24657

[6] A. Abulencia et al. [CDF Collaboration], J. Phys. G 34, 2457 (2007) [arXiv:hep-ex/0508029].

[7] V. M. Abazov et al. [D0 Collaboration], Nucl. Instrum. Meth. A 565, 463 (2006) [arXiv:physics/0507191].

[8] V. M. Abazov et al. [D0 Collaboration], arXiv:0803.2137 [hep-ex], accepted by Phys. Rev. Lett.

[9] M. Goncharov et al., Nucl. Instrum. Meth. A 565, 543 (2006) [arXiv:physics/0512171].

[10] A. Abulencia et al. [CDF Collaboration], Phys. Rev. Lett. 97, 171802 (2006) [arXiv:hep-ex/0605101].

[11] J. Abdallah et al. [DELPHI Collaboration], Eur. Phys. J. C 38, 395 (2005) [arXiv:hep-ex/0406019]; P. Achard et al. [L3 Collaboration], Phys. Lett. B 587, 16 (2004) [arXiv:hep-ex/0402002].

[12] V. M. Abazov et al. [D0 Collaboration], Phys. Rev. Lett. 95, 161602 (2005) [arXiv:hep-ex/0506063].

[13] V. M. Abazov et al. [D0 Collaboration], DØ note 4336 (2004), unpublished.

[14] T. Aaltonen et al. [CDF Collaboration], arXiv:0801.4806 [hep-ex].

[15] V. M. Abazov et al. [D0 Collaboration], Phys. Rev. Lett. 100, 131801 (2008) [arXiv:0712.0599 [hep-ex]].

[16] M. Kober, B. Koch and M. Bleicher, Phys. Rev. D 76, 125001 (2007) [arXiv:0708.2368 [hep-ph]] and references therein. 